\title{
EFEITO DE SUPRESSÃO NAS VIAS AUDITIVAS: UM ESTUDO COM OS POTENCIAIS DE MÉDIA E LONGA LATÊNCIA
}

\author{
Effect of suppression in the auditory pathways: \\ a study with middle and long latency potentials
}

\author{
Mariana Buncana Simões ${ }^{(1)}$, Renata Rezende de Souza ${ }^{(2)}$, Eliane Schochat ${ }^{(3)}$
}

\begin{abstract}
RESUMO
Objetivo: avaliar o efeito de supressão no Potencial Evocado Auditivo de Média e Longa Latência em indivíduos sem alterações auditivas, com presença de emissões otoacústicas (EOA) transientes em ambas as orelhas. Métodos: foram avaliados 25 indivíduos (50 orelhas) de 18 a 30 anos de idade, de ambos os sexos, utilizando-se os Potenciais Evocados Auditivos de Média e Longa latência sem e com ruído branco contralateral. Os sujeitos não apresentavam queixa relacionada ao Processamento Auditivo. Foram verificados e comparados os valores numéricos das latências e amplitudes das ondas na avaliação convencional sem ruído e com ruído contralateral. Resultados: a média aritmética da amplitude de resposta na situação com ruído (mascaramento) reduziu em todas as posições (C3/ A1, C4/A1, C3/A2 e C4/A2), porém estes achados foram estatisticamente significantes nas posições C4/A1, C3/A2 e C4/A2. Conclusão: a diminuição da amplitude pode ter relação com o número de sinapses realizadas. $O$ efeito de supressão na amplitude pode estar relacionado à ação do sistema eferente que suprime o número de sinapses neuronais.
\end{abstract}

DESCRITORES: Potenciais Evocados; Audiologia; Testes Auditivos

\section{INTRODUÇÃO}

A avaliação da função auditiva pode ser realizada por inúmeros testes subjetivos e objetivos, que buscam informações relacionadas à audição periférica e central. Para avaliação das vias auditivas até o córtex auditivo têm sido utilizados testes ele-

(1) Fonoaudióloga; Centro de Docência e Pesquisa do Departamento de Fisioterapia, Fonoaudiologia e Terapia Ocupacional da Faculdade de Medicina da Universidade de São Paulo, USP, São Paulo, SP; Mestranda da Faculdade de Medicina da Universidade de São Paulo do Programa: Ciências da Reabilitação.

(2) Fonoaudióloga; Centro de Docência e Pesquisa do Departamento de Fisioterapia, Fonoaudiologia e Terapia Ocupacional da Faculdade de Medicina da Universidade de São Paulo, USP, São Paulo, SP; Mestranda da Faculdade de Medicina da Universidade de São Paulo do Programa: Ciências da Reabilitação.

(3) Fonoaudióloga; Professor Associado Doutor do Curso de Fonoaudiologia da Universidade de São Paulo, USP, São Paulo, SP; Doutora em Psicolínguística pela Faculdade de Filosofia, Letras e Ciências Humanas da Universidade de São Paulo; Livre Docente pelo Departamento de Fisioterapia, Fonoaudiologia e Terapia Ocupacional da Faculdade de Medicina da Universidade de São Paulo. trofisiológicos ou também denominados Potenciais Evocados Auditivos. A classificação mais utilizada dos Potenciais Evocados Auditivos baseia-se no período de latência das respostas, que é o tempo transcorrido desde a apresentação do estímulo até o aparecimento das mesmas, que são analisadas em milissegundos.

Evidências anatômicas e fisiológicas sustentam a função interdependente das duas orelhas coordenadas através das vias neurais eferentes, que ligam um lado do sistema auditivo ao outro, por meio dos componentes do sistema olivococlear. O Sistema Olivococlear Medial recebe entrada predominantemente do núcleo coclear contralateral, e as projeções das fibras mediais eferentes são predominantemente cruzadas ${ }^{1}$. O efeito eferente mais conhecido é a diminuição do potencial de ação do nervo auditivo. Esse potencial é produzido por descargas sincrônicas das fibras auditivas aferentes radiais, mielinizadas, que inervam as células ciliadas internas, sem contribuição das fibras auditivas aferentes espirais, não mielinizadas, que inervam as células ciliadas externas ${ }^{2}$. $\mathrm{O}$ trato olivococlear medial age sobre os movimentos das células 
ciliadas externas (CCE) provocando uma hiperpolarização através da liberação de acetilcolina na fenda sináptica ${ }^{3,4}$. Esta hiperpolarização ocorre em oposição à despolarização, induzida naturalmente pelos estímulos sonoros e é evidenciada pela redução da amplitude das respostas com o uso de uma estimulação acústica na orelha contralateral ${ }^{5,6}$. O Sistema Olivocolear Eferente Medial atua como sistema de proteção contra ruídos intensos e na atenção seletiva ${ }^{7}$.

As respostas auditivas de média latência (PEAML) e de longa latência (PEALL) consistem em uma série de ondas positivas e negativas ${ }^{8}$. O Potencial Evocado Auditivo de Média Latência (PEAML), primeiramente descrito por Geisler e cols. em $1958^{9}$, é composto por uma série de ondas observadas num intervalo entre 15 e 80 milessegundos após o início do estímulo auditivo ${ }^{10}$. A captação desses potenciais reflete a atividade cortical envolvida nas habilidades de audição primária (reconhecimento, discriminação e figura-fundo) e não primária (atenção seletiva, sequência auditiva e integração auditiva/visual) ${ }^{11}$.

A amplitude da onda Na-Pa do PEAML em sujeitos normais é simétrica, ou seja, eletrodos colocados no Lobo Temporal direito e esquerdo devem obter respostas similares ${ }^{12}$. A análise das ondas deve ser realizada em uma observação comparativa entre os hemisférios e entre as orelhas (direita e esquerda) e intra-sujeito para a determinação de normalidade, já que este potencial ainda necessita de mais estudos com relação à sensibilidade $e$ especificidade ${ }^{12}$. No PEAML é possível reconhecer quatro ondas positivas e três negativas, mas, usualmente, são analisadas apenas as ondas $\mathrm{Na}(18 \mathrm{~ms})$ e Pa (30ms), já que são as maiores em amplitude e mais estáveis que as outras.

Já os Potenciais de Longa Latência (PLL) são menos afetados pelas propriedades físicas do estímulo e mais afetados pelo uso funcional que o indivíduo faz do estímulo. A resposta pode ser determinada menos pela frequência ou intensidade do estímulo e mais pela atenção ao estímulo, ou uma mudança de estímulo. Os potencias de Longa Latência refletem principalmente a atividade do tálamo e córtex; estruturas responsáveis por funções de discriminação, integração e atenção auditivas ${ }^{13}$. O potencial evocado que ocorre no cérebro em resposta a um evento interno, como a percepção ou cognição, é denominado potencial relacionado a evento, também considerado potencial endógeno ${ }^{14}$. Este é o potencial cognitivo P300 ${ }^{15}$. O potencial cognitivo P300 tem sido usado como um procedimento objetivo para avaliar a função cognitiva cerebral.
Descrito originalmente por Sutton e cols. (1965) ${ }^{14}$, a resposta do P300 depende da atenção e da discriminação das diferenças de estímulos. Esse potencial aparece quando um estímulo inesperado ocorre dentro de uma série de estímulos esperados. Ele ocorre aproximadamente $300 \mathrm{~ms}$ após o início do estímulo (valores de 250 a 500ms), com amplitude variando de 7 a 25 microVolts ${ }^{14}$.

O complexo N1P2 também é um potencial de longa latência, porém é exógeno, ou seja, é relacionado às características do estímulo. A resposta eletrofisiológica N1 é obtida entre 80 e 110 milisegundos após estimulação acústica e P2 de 150 e 200 milisegundos ${ }^{14}$.

Em um estudo conduzido em 2003, autores ${ }^{16}$ examinaram o efeito do mascaramento contralateral com ruído branco em potenciais auditivos corticais (N1 e P2) e verificaram uma diminuição significante na amplitude do N1 na presença do ruído branco na intensidade de $75 \mathrm{~dB}$ NA, o que não foi verificado para o P2. Eles sugeriram que este efeito pode ter ocorrido por causa do sistema auditivo eferente.

Estudiosos ${ }^{17}$ também mostraram que a estimulação contralateral reduz a amplitude dos Potenciais Evocados Auditivos (PEAs) e que existem duas prováveis causas para essa redução:

1. Atenção Dividida

2. Efeito da via Eferente

Esse estudo foi realizado utilizando desde os potenciais auditivos médios até os mais tardios na presença e ausência de estimulação contralateral para analisar o comportamento do efeito de supressão (diminuição das respostas) durante toda a via auditiva.

Deste modo, o objetivo foi avaliar o efeito de supressão do Potencial Evocado Auditivo de Média e Longa Latência em indivíduos sem alterações auditivas com presença de EOA transientes em ambas as orelhas.

\section{MÉTODOS}

Foram avaliados 25 indivíduos ( 50 orelhas) de 18 a 30 anos de idade (média de 25,3 anos de idade) de ambos os sexos (68\% do sexo feminino), com Emissões Otoacústicas Transientes presentes em ambas as orelhas (excluindo assim, qualquer comprometimento de orelha média ou perda auditiva que pudesse comprometer o exame), utilizandose os Potenciais Evocados Auditivos de Média e Longa latência, sem e com ruído branco contralateral. A pesquisa foi realizada no Centro de Docência e Pesquisa do Departamento de Fisioterapia, Fonoaudiologia e Terapia Ocupacional da Faculdade de Medicina da Universidade de São Paulo, durante o ano de 2005. Os sujeitos avaliados assinaram um 
termo de consentimento livre e esclarecido. Apresentaram audiometria tonal, vocal e imitanciometria dentro dos padrões de normalidade e não apresentavam queixas relacionadas ao Processamento Auditivo.

A pesquisa dos Potenciais foi realizada por meio de um computador com o programa Bio-logic systems corp. em sala silenciosa com o uso de fones TDH39. Para iniciar a avaliação eletrofisiológica, era necessário que os eletrodos apresentassem impedância menor que 5KW. O exame foi realizado com o indivíduo sentado confortavelmente com os olhos fechados (para a eliminação do artefato causado pela movimentação ocular). Para o MLR foi utilizado o click não-linear a $70 \mathrm{~dB}$ e ruído branco contralateral a $70 \mathrm{~dB}$. Os eletrodos foram dispostos nas mastóides (A1 e A2), nos lobos temporais ou região coronal esquerda e direita $(C 3, C 4$ respectivamente) e na fronte ( $\mathrm{A}$ - terra ou comum). Os estímulos foram apresentados por fones e as respostas gravadas duas vezes em cada condição (C3A1, C4A1, C3A2 e C4A2) para aumentar a fidedignidade das mesmas. A latência foi medida no pico da onda e a amplitude foi medida utilizando-se como base a onda Na. Para avaliar o PLL, os eletrodos ativos foram colocados em $\mathrm{Cz}$. Os eletrodos de referência foram posicionados nas mastóides direita e esquerda e o eletrodo terra foi colocado na posição Fpz. Para a realização do P300 foi utilizado estímulo tone burst na frequência de $2 \mathrm{KHz}$ para o estímulo raro, apresentado randomicamente na probabilidade de $20 \%$, mesclado ao estímulo tone burst frequente em $1 \mathrm{kHz}$ apresentado na probabilidade de $80 \%$, intensidade moderada de $70 \mathrm{~dB}$, velocidade de um estímulo por segundo. Foi solicitado que os indivíduos identificassem o estímulo raro, contando-o mentalmente.

A análise foi baseada:

1 - Nos valores numéricos das latências e amplitudes das ondas na avaliação convencional sem ruído;

2 - Nos valores numéricos das latências e amplitudes das ondas na avaliação convencional com ruído;

3-Na análise dos valores obtidos nas avaliações na presença e ausência do ruído contralateral.

O estudo foi submetido à Comissão de Ética para Análise de Projetos de Pesquisa do Hospital das Clínicas da Faculdade de Medicina da Universidade de São Paulo (Protocolo de Pesquisa número 227/05).

Foram utilizados os testes T-student pareado (paramétrico) e Wilcoxon (não paramétrico). Para complementar a análise descritiva, foi utilizada a técnica de Intervalo de Confiança para média. Para este trabalho foi definido um nível de significância de $0,05(5 \%)$. Todos os intervalos de confiança construídos ao longo do trabalho foram construídos com $95 \%$ de confiança estatística.

\section{RESULTADOS}

Foram comparados os resultados Com e Sem Ruído para o PEAML. A começar pela posição C3/A1 (Tabelas 1, 2, 3 e 4).

As diferenças encontradas entre as condições Sem e Com Mascaramento na posição C3/A1, não foram estatisticamente significantes, embora a amplitude apresente média numérica maior na condição Sem Mascaramento do que na Com Mascaramento.

Foi averiguado que, para a latência de $\mathrm{Na}$ e Amplitude $\mathrm{Na}-\mathrm{Pa}$, as diferenças entre Sem e Com Mascaramento são estatisticamente significantes. Para latência de $\mathrm{Na}$ o maior resultado (média, mediana e quartis) encontrou-se na condição Com Mascaramento. No caso de amplitude, o maior resultado foi encontrado na condição Sem Mascaramento.

Em nenhum momento na posição de C3/A2, as diferenças entre Sem e Com Mascaramento foram estatisticamente significantes. Pôde-se verificar que na latência de $\mathrm{Pa}$ o $\mathrm{p}$-valor estava muito próximo do limite aceitável, assim, pode-se dizer que existe uma tendência à significância. Entretanto, cabe ressaltar que a média aritmética da amplitude na condição Sem Mascaramento é maior do que Com Mascaramento.

Para finalizar, foi comparado o mascaramento para a posição de C4/A2.

Na posição de C4/A2, somente existe diferença estatisticamente significante para a amplitude, onde os resultados (média, mediana e quartis) de Sem Mascaramento são maiores do que Com Mascaramento.

As diferenças médias encontradas entre as condições Sem e Com Ruído para o P300 não foram consideradas estatisticamente significantes, embora se observou que a situação de Sem Ruído possui maior média para amplitude e latência em ambas as orelhas.

Existe diferença média estatisticamente significante entre as condições Sem e Com Ruído para a amplitude tanto na orelha direita quanto na esquerda, sendo que a amplitude medida na condição Sem Ruído apresenta a maior média.

Para P2, somente na amplitude da orelha esquerda é que a diferença entre as condições Sem e Com Ruído é considerada estatisticamente significante, sendo que a condição Sem Ruído possui a maior média para ambas as orelhas. 
Tabela 1 - Latência e Amplitude do PEAML na posição C3/A1 Sem e Com Mascaramento

\begin{tabular}{lcccccc}
\hline \multirow{2}{*}{ C3/A1 } & \multicolumn{2}{c}{ Na (latência) } & \multicolumn{2}{c}{ Pa(latência) } & \multicolumn{2}{c}{ Amp } \\
\cline { 2 - 7 } & Sem & Com & Sem & Com & Sem & Com \\
\hline Média & 18,80 & 18,91 & 33,31 & 34,10 & 2,41 & 2,32 \\
Mediana & 18,72 & 18,33 & 33,54 & 33,15 & 1,84 & 1,54 \\
Desvio Padrão & 1,41 & 2,08 & 2,51 & 3,08 & 2,35 & 2,36 \\
Quartil 1 & 17,94 & 17,55 & 31,98 & 32,37 & 1,48 & 1,03 \\
Quartil 3 & 19,89 & 19,11 & 34,71 & 36,27 & 2,44 & 2,58 \\
Tamanho & 25 & 25 & 25 & 25 & 25 & 25 \\
IC & 0,55 & 0,82 & 0,98 & 1,21 & 0,92 & 0,92 \\
\hline p-valor & \multicolumn{3}{c}{0,935} & 0,212 & \multicolumn{3}{c}{0,375} \\
\hline
\end{tabular}

IC: Intervalo de Confiança (mostra a variação da média segundo uma probabilidade estatística).

$\mathrm{Na}$ : Onda $\mathrm{Na}$

$\mathrm{Pa}$ : Onda $\mathrm{Pa}$

Amp: Amplitude

Tabela 2 - Latência e Amplitude do PEAML na posição C4/A1 Sem e Com Mascaramento

\begin{tabular}{lcccccc}
\hline \multirow{2}{*}{ C4/A1 } & \multicolumn{2}{c}{ Na(latência) } & \multicolumn{2}{c}{ Pa(latência) } & \multicolumn{3}{c}{ Amp } \\
\cline { 2 - 7 } & Sem & Com & Sem & Com & Sem & Com \\
\hline Média & 19,02 & 19,84 & 33,54 & 34,12 & 2,37 & 2,19 \\
Mediana & 18,72 & 19,5 & 33,54 & 33,93 & 1,82 & 1,35 \\
Desvio Padrão & 1,52 & 2,32 & 2,07 & 2,29 & 2,46 & 2,55 \\
Quartil 1 & 18,33 & 18,72 & 32,76 & 32,76 & 1,15 & 0,83 \\
Quartil 3 & 19,5 & 19,89 & 34,71 & 35,49 & 2,79 & 2,33 \\
Tamanho & 25 & 25 & 25 & 25 & 25 & 25 \\
IC & 0,60 & 0,91 & 0,81 & 0,90 & 0,97 & 1,00 \\
\hline p-valor & \multicolumn{3}{c}{$0,032^{*}$} & \multicolumn{3}{c}{0,254} \\
\hline
\end{tabular}

* $p$-valores considerados estatisticamente significantes perante o nível de significância adotado.

IC: Intervalo de Confiança (mostra a variação da média segundo uma probabilidade estatística).

$\mathrm{Na}$ : Onda $\mathrm{Na}$

$\mathrm{Pa}$ : Onda $\mathrm{Pa}$

Amp: Amplitude

Tabela 3 - Latência e Amplitude do PEAML na posição C3/A2 Sem e Com Mascaramento

\begin{tabular}{lcccccc}
\hline \multirow{2}{*}{ C3/A2 } & \multicolumn{2}{c}{ Na(latência) } & \multicolumn{2}{c}{ Pa(latência) } & \multicolumn{2}{c}{ Amp } \\
\cline { 2 - 7 } & Sem & Com & Sem & Com & Sem & Com \\
\hline Média & 18,58 & 18,64 & 33,56 & 34,18 & 2,37 & 2,61 \\
Mediana & 18,72 & 18,72 & 32,76 & 33,54 & 1,95 & 1,99 \\
Desvio Padrão & 0,94 & 1,34 & 2,52 & 2,52 & 1,69 & 2,65 \\
Quartil 1 & 18,33 & 17,94 & 31,59 & 31,98 & 1,55 & 0,95 \\
Quartil 3 & 19,11 & 19,5 & 35,88 & 36,66 & 2,82 & 3,01 \\
Tamanho & 25 & 25 & 25 & 25 & 25 & 25 \\
IC & 0,37 & 0,53 & 0,99 & 0,99 & 0,66 & 1,04 \\
\hline p-valor & \multicolumn{3}{c}{0,909} & \multicolumn{3}{c}{$0,081 \#$} \\
\hline
\end{tabular}

\# p-valores que por estarem próximos do limite de aceitação, são considerados que tendem a ser significantes.

IC: Intervalo de Confiança (mostra a variação da média segundo uma probabilidade estatistica).

$\mathrm{Na}:$ Onda $\mathrm{Na}$

$\mathrm{Pa}$ : Onda $\mathrm{Pa}$

Amp: Amplitude 
Tabela 4 - Latência e Amplitude do PEAML na posição C4/A2 Sem e Com Mascaramento

\begin{tabular}{lcccccc}
\hline \multirow{2}{*}{ C4/A2 } & \multicolumn{2}{c}{ Na(latência) } & \multicolumn{2}{c}{ Pa(latência) } & \multicolumn{3}{c}{ Amp } \\
\cline { 2 - 7 } & Sem & Com & Sem & Com & Sem & Com \\
\hline Média & 18,78 & 18,39 & 33,35 & 33,54 & 2,06 & 1,59 \\
Mediana & 18,72 & 18,33 & 32,76 & 33,54 & 1,89 & 1,09 \\
Desvio Padrão & 1,86 & 2,11 & 2,34 & 2,86 & 1,57 & 1,47 \\
Quartil 1 & 17,55 & 17,16 & 31,98 & 31,2 & 1,06 & 0,93 \\
Quartil 3 & 19,5 & 19,11 & 34,71 & 36,27 & 2,37 & 1,77 \\
Tamanho & 25 & 25 & 25 & 25 & 25 & 25 \\
IC & 0,73 & 0,83 & 0,92 & 1,12 & 0,61 & 0,58 \\
\hline p-valor & \multicolumn{3}{c}{0,229} & 0,742 & \multicolumn{3}{c}{$0,002^{*}$} \\
\hline
\end{tabular}

* $p$-valores considerados estatisticamente significantes perante o nível de significância adotado. IC: Intervalo de Confiança (mostra a variação da média segundo uma probabilidade estatistica).

$\mathrm{Na}$ : Onda $\mathrm{Na}$

$\mathrm{Pa}$ : Onda $\mathrm{Pa}$

Amp: Amplitude

\section{DISCUSSÃO}

Uma forma de se avaliar o efeito de supressão das vias eferentes auditivas é por meio da introdução de estímulo contralateral à orelha testada, esperando assim a diminuição das respostas obtidas quando comparadas com as respostas sem estímulo competitivo.

Este efeito já foi intensamente estudado através das Emissões Otoacústicas (EOA) ${ }^{18-20}$. As vias eferentes, por meio do sistema olivococlear eferente medial, são responsáveis pela modulação inibitória das contrações rápidas das células ciliadas externas através da produção de contrações lentas nessas mesmas células, atenuando o processo de amplificação coclear.

Estudos realizados previamente com os Potenciais de Média Latência não apresentaram diferenças significantes no desempenho dos indivíduos do sexo masculino e feminino ${ }^{21,22}$.

$\mathrm{Na}$ análise das respostas do PEAML (Tabelas 1, 2, 3 e 4), houve redução na média aritmética da amplitude de resposta na situação com ruído (mascaramento) em todas as posições (C3/A1, C4/A1, C3/A2 e C4/A2), porém estes achados só foram estatisticamente significantes nas posições C4/A1, C3/A2 e C4/A2. Em estudo conduzido por Eisencraft e cols. em $2006{ }^{17}$, no qual foi utilizada música como efeito mascarante no PEAML, foi verificada diminuição da amplitude em todas as posições dos eletrodos, corroborando com os achados desse estudo. A amplitude das vias contralaterais (C3A2 e C4A1) deveria ser maior que a amplitude das ipsilaterais (C3A1 e C4A2) devido ao fato de que o número de fibras da via contralateral auditiva é maior que na via ipsilateral ${ }^{2}$
Porém, esta diferença não pôde ser observada quando se examinam os resultados do PEAML, talvez devido à grande variabilidade, que pode ser encontrada entre os indivíduos tanto na amplitude quanto na latência deste potencial, uma vez que ainda não há padronização das respostas do PEAML ${ }^{12}$.

É importante destacar que o escasso número de publicações correlacionando o efeito de supressão com os Potenciais de Média e Longa Latência dificulta a análise e discussão deste estudo.

A amplitude da resposta do P300 (Tabela 5) diminuiu na situação com ruído mascarante em ambas as orelhas, apesar do resultado não ser estatisticamente significante. A amplitude diminuiu de forma significante em ambas orelhas, medindo-se a onda N1 (Tabela 6). Na medida da onda P2 (Tabela 7) houve diminuição da amplitude da resposta nas duas orelhas, mas apenas a diminuição da amplitude na orelha esquerda foi estatisticamente significante. Autores ${ }^{16}$ verificaram diminuição da amplitude de resposta do N1 na apresentação de ruído branco contralateral, mas não a diminuição da amplitude da onda P2. A ocorrência da atenuação na amplitude no complexo N1P2 pode ser devido a um processo inibitório, causado pelo desvio da atenção provocado pelo ruído, muito embora este potencial seja considerado exógeno e pré-atencional, isto é, insensível a operações cognitivas e atencionais. Outros estudos também encontraram redução na amplitude da onda, e os autores associaram este fato a um efeito da atenção ${ }^{23-25}$.

$O$ efeito do mascaramento contralateral nas ondas do PEAML e do PLL pode estar sendo mediado pelo efeito eferente coclear, assim como ocorre diminuição nas emissões otoacústicas na 
Tabela 5 - Latência e Amplitude do P300 Sem e Com Mascaramento

\begin{tabular}{|c|c|c|c|c|c|c|c|c|}
\hline \multirow{3}{*}{ P300 } & \multicolumn{4}{|c|}{ Orelha Direita } & \multicolumn{4}{|c|}{ Orelha Esquerda } \\
\hline & \multicolumn{2}{|c|}{ latência } & \multicolumn{2}{|c|}{ amplitude } & \multicolumn{2}{|c|}{ latência } & \multicolumn{2}{|c|}{ Amplitude } \\
\hline & Sem & Com & Sem & Com & Sem & Com & Sem & Com \\
\hline Média & 324,48 & 320,68 & 10,82 & 9,80 & 313,64 & 312,64 & 9,56 & 8,59 \\
\hline Mediana & 328 & 321 & 9,46 & 8,04 & 324 & 312 & 9,71 & 6,29 \\
\hline Desvio Padrão & 35,99 & 41,73 & 5,14 & 5,73 & 46,61 & 44,28 & 3,55 & 5,60 \\
\hline Mínimo & 257 & 247 & 4,11 & 2,75 & 221 & 237 & 3,67 & 2,81 \\
\hline Máximo & 377 & 413 & 25,21 & 23,55 & 385 & 395 & 15,11 & 21,22 \\
\hline Tamanho & 25 & 25 & 25 & 25 & 25 & 25 & 25 & 25 \\
\hline IC & 14,11 & 16,36 & 2,01 & 2,25 & 18,27 & 17,36 & 1,39 & 2,20 \\
\hline$p$-valor & \multicolumn{2}{|c|}{0,590} & \multicolumn{2}{|c|}{0,241} & \multicolumn{2}{|c|}{0,872} & \multicolumn{2}{|c|}{0,236} \\
\hline
\end{tabular}

IC: Intervalo de Confiança (mostra a variação da média segundo uma probabilidade estatistica).

P300: onda P300

Tabela 6 - Latência e Amplitude do N1 Sem e Com Mascaramento

\begin{tabular}{lcccccccc}
\hline & \multicolumn{4}{c}{ Orelha Direita } & \multicolumn{4}{c}{ Orelha Esquerda } \\
\cline { 2 - 9 } N1 & \multicolumn{2}{c}{ latência } & \multicolumn{2}{c}{ amplitude } & \multicolumn{2}{c}{ latência } & \multicolumn{2}{c}{ amplitude } \\
\cline { 2 - 10 } & Sem & Com & Sem & Com & Sem & Com & Sem & Com \\
\hline Média & 88,92 & 89,00 & 3,82 & 3,42 & 88,80 & 89,08 & 3,94 & 3,04 \\
Mediana & 88 & 90 & 3,57 & 3,21 & 89 & 89 & 3,74 & 2,9 \\
Desvio Padrão & 9,22 & 8,11 & 1,44 & 1,28 & 8,53 & 9,62 & 1,89 & 1,34 \\
Mínimo & 65 & 66 & 1,49 & 1,3 & 75 & 55 & 1,04 & 0,26 \\
Máximo & 102 & 106 & 6,75 & 6,17 & 104 & 102 & 7,97 & 6,11 \\
Tamanho & 25 & 25 & 25 & 25 & 25 & 25 & 25 & 25 \\
IC & 3,62 & 3,18 & 0,57 & 0,50 & 3,35 & 3,77 & 0,74 & 0,53 \\
\hline p-valor & \multicolumn{3}{c}{0,942} & \multicolumn{2}{c}{$0,015^{*}$} & \multicolumn{2}{c}{0,905} & $<, 001^{*}$ \\
\hline
\end{tabular}

* $p$-valores considerados estatisticamente significantes perante o nível de significância adotado. IC: Intervalo de Confiança (mostra a variação da média segundo uma probabilidade estatistica).

N1: Onda N1

Tabela 7 - Latência e Amplitude do P2 Sem e Com Mascaramento

\begin{tabular}{lcccccccc}
\hline & \multicolumn{4}{c}{ Orelha Direita } & \multicolumn{3}{c}{ Orelha Esquerda } \\
\cline { 2 - 9 } P2 & \multicolumn{2}{c}{ latência } & \multicolumn{2}{c}{ amplitude } & \multicolumn{2}{c}{ latência } & \multicolumn{2}{c}{ amplitude } \\
\cline { 2 - 9 } & Sem & Com & Sem & Com & Sem & Com & Sem & Com \\
\hline Média & 168,92 & 150,88 & 3,49 & 3,20 & 158,32 & 153,88 & 3,51 & 2,65 \\
Mediana & 156 & 152 & 3,46 & 3 & 154 & 154 & 3,42 & 2,48 \\
Desvio Padrão & 46,56 & 35,17 & 1,63 & 1,42 & 16,64 & 36,47 & 2,02 & 1,46 \\
Mínimo & 103 & 2,03 & 0,87 & 0,87 & 125 & 2,99 & 0,2 & 0,51 \\
Máximo & 321 & 197 & 6,76 & 6 & 192 & 192 & 8,01 & 6,13 \\
Tamanho & 25 & 25 & 25 & 25 & 25 & 25 & 25 & 25 \\
IC & 18,25 & 13,79 & 0,64 & 0,56 & 6,52 & 14,30 & 0,79 & 0,57 \\
\hline p-valor & \multicolumn{3}{c}{0,108} & \multicolumn{2}{c}{0,125} & \multicolumn{2}{c}{0,544} & \multicolumn{2}{c}{$<0,001^{*}$} \\
\hline
\end{tabular}

* $\mathrm{p}$-valores considerados estatisticamente significantes perante o nível de significância adotado.

IC: Intervalo de Confiança (mostra a variação da média segundo uma probabilidade estatistica).

P2: Onda P2 
presença de um ruído na orelha contralateral, que é mediada pela via eferente. As células ciliadas externas da cóclea contralateral (a que está recebendo o ruído) são pertencentes à via eferente que vai da cóclea até o complexo olivar e fazem parte do Sistema Eferente Medial ${ }^{26}$. Apesar das inúmeras pesquisas para determinar a ação das vias auditivas eferentes sobre a audição humana, os mecanismos fisiológicos de interação dos sistemas eferente e aferente ainda não foram bem esclarecidos.

\section{CONCLUSÃO}

Foi constatada redução na média aritmética da amplitude de resposta na situação com ruído em todas as posições dos eletrodos no PEAML. A amplitude da resposta do P300 e das ondas N1 e P2 diminuiu na situação com ruído em ambas as orelhas. A diminuição da amplitude pode ter relação com o número de sinapses realizadas. $O$ efeito de supressão na amplitude pode estar relacionado à ação do sistema eferente que suprime o número de sinapses neuronais.

\begin{abstract}
Purpose: to evaluate the suppression effect of the Middle Latency Response (MLR) and Long Latency Potential on normal hearing individuals. Methods: twenty-five individuals of both genders between 18 and 30-year old were evaluated (50 ears), with presence of OAE in both ears (thus, excluding any middle ear problem or hearing loss that could compromise the final evaluation). The individuals did not present any complain on the Auditory Processing. We used MLR and P300 with and with no contralateral white noise. The latency and amplitude of the waves were evaluated (with and with no noise) and the results were compared. Results: we found reduction in the median amplitude in the situation with noise (masking) in all positions (C3/A1, C4/A1, C3/A2 and C4/A2). However, these findings had no statistically significant difference in the positions $C 3 / A 1$. Conclusion: the effect of the suppression on the amplitude could be linked to the efferent system that decreases the number of neuronal synapses.
\end{abstract}

KEYWORDS: Evoked Potencials; Audiology; Hearing Tests

\section{REFERÊNCIAS}

1. Warr WB, Guinan JJ. Efferent innervation of the organ of Corti, two separate systems. Brain Res. 1979; 173(1):152-5.

2. Veuillet E, Collet L, Duclaux R. Effect of contralateral acoustic stimulation on active cochlear micromechanical properties in humans subjects: dependence on stimulus variables. J Neurophysiol. 1991; 65(3):724-35.

3. Sahley TL, Nodar RH, Musiek FE. Efferent auditory system, structure and function. 1. ed. San Diego: Singular; 1997. p.1-23.

4. Funchs $P$. The synaptic physiology of cochlear hair cells. Audiol Neurootol. 2002; 7: 40-4.

5. De Ceulaer G, Yperman M, Daemers K, Van Driessche K, Somers T, Officierss FE, Govaerts PJ. Contralateral suppression of transient evoked otoacoustic emission: normative data for a clinical test set-up. Otol Neurotol. 2001; 22(3):350-5.

6. James AL, Mount RJ, Harrison RV. Contralateral suppression of DPOAE measured in real time. Clin Otolaryngol. 2002; 27(2):106-12.
7. Rasmussen GL. The olivary penducle and other fiber projections of the superior olivary complex. $J$ Comp Neurol. 1946; 84:141-219.

8. Musiek FE, Lee WW. Potenciais auditivos de média e longa latência. In: Musiek FE, Rintelmann WF. Perspectivas atuais em avaliação auditiva. Barueri: Manole; 2001. p. 239-67.

9. Geisler CD, Frishkopf LS, Rosenblith WA. Extracranial responses to acoustic clicks in man. Science.1958; 128:1210-1.

10. Musiek F, Charette L, Kelly T, Lee WW, Musiek E. Hit and false-positive rates for the middle latency response in patients with central nervous system involvement. J Am Acad Audiol. 1999; 10(3):124-32.

11. Kraus N, Kileny P, Mcgee T. Middle Latency Auditory Evoked Potentials. In: Katz J. organizador. Handbook of clinical auditory. 4. ed. Baltimore: Williams \& Wilkins; 1994. p. 387-405.

12. Schochat E, Rabelo CM, Almeida RC. Sensitividade e especificidade do potencial de latência média. Rev Bras Otorrinolaringol. 2004; 70(3):353-8. 
13. Kraus N, Mcgee T. Potenciais evocados auditivos de longa latência. In: Katz J. Tratado de audiologia clínica. São Paulo: Manole; 1999. p. 403-20. 14. Sutton S, Braren SM, Zubin J, John ER. Evokedpotential correlates of stimulus uncertainty. Science. 1965; 150(700):1187-8.

15. Picton TW. The P300 wave of the human event-related potential. J Clin Neurophysiol. 1992; 9(4):456-79.

16. Salo SK, Lang AH, Salmivalli AJ, Johansson Rk, Peltola MS. Contralateral white noise masking affects auditory N1 and P2 waves differently. Int J Psychophysiol. 2003; 17(4):189-94.

17. Eisencraft T, Miranda MF, Schochat E. Comparação dos potenciais de latência média com ou sem estímulo musical. Rev Bras Otorrinolaringol. 2006; 72(4):465-9.

18. Berlin CA, Hood LJ, Hurley A, Wen $H$. Contralateral suppression of otoacoustic emissions an index of the function of the medial olivocochlear system. Otolaryngol Head Neck Surg. 1994; 110(1):3-21.

19. Berlin CA, Hood LJ, Hurley A, Wen H. Hearing aids: only for hearing impaired patients with abnormal otoacoustic emissions. In: Berlin $\mathrm{Cl}$. Hearing cells and hearing aids. San Diego: Singular Publishing Group; 1996. p 3-27.

20. Lopes FilhoOC, Carlos R. Emissões otoacústicas. In: Lopes Filho OC. Tratado de fonoaudiologia. São Paulo: Tecmedd; 2005. p. 207-22.

21. Schochat, E. Resposta de latência média em crianças e adolescentes normo-ouvintes. Pró-Fono. 2003; 15(1):65-74.

22. Schochat E, Rabelo CM, Loreti RC de A. Sensitividade e especificidade do potencial de média latência. Rev Bras Otorrinolaringol. 2004; 70(3):353-8.

23. Jerger K, Biggins C, Fein G. P50 suppression is not affected by attentional manipulations. Biol Psychiatr. 1992; 31(4):365-77.

24. Guterman $Y$, Josiassen RC, Bashore TRJ. Attentional influence on the P50 component of the auditory event-related brain potential. Int $\mathrm{J}$ Psychophysiol. 1992; 12:197-209.

25. Guterman Y, Josiassen RC. Sensory gating deviance in schizophrenia in the context of task related effects. Int J Psychophysiol. 1994; 18:1-12. 26. Klinke R, Galley N. Efferent innervation of vestibular and auditory receptors. Physiol Rev. 1974; 54(2):316-57.

DOI: 10.1590 / S1516-18462008005000011

RECEBIDO EM: 28/09/2007

ACEITO EM: 26/05/2008

Endereço para correspondência:

Mariana Buncana Simões

Rua Bourbon, 84

São Paulo - SP

CEP: 04663-160

E-mail: simoesmari@ hotmail.com 